\title{
EchoGéo
}

$53 \mid 2020$

Dénominations plurielles. Quand les noms de lieux se concurrencent

\section{Nommer les biens du Patrimoine mondial : processus de patrimonialisation et réinvention toponymique}

\section{Christophe Gauchon}

\section{(2) OpenEdition \\ Journals}

Édition électronique

URL : https://journals.openedition.org/echogeo/19973

DOI : 10.4000/echogeo.19973

ISSN : 1963-1197

Éditeur

Pôle de recherche pour l'organisation et la diffusion de l'information géographique (CNRS UMR 8586)

Référence électronique

Christophe Gauchon, « Nommer les biens du Patrimoine mondial : processus de patrimonialisation et réinvention toponymique », EchoGéo [En ligne], 53 | 2020, mis en ligne le 30 septembre 2020, consulté le 10 août 2021. URL : http://journals.openedition.org/echogeo/19973 ; DOI : https://doi.org/10.4000/ echogeo.19973

Ce document a été généré automatiquement le 10 août 2021.

EchoGéo est mis à disposition selon les termes de la licence Creative Commons Attribution - Pas d'Utilisation Commerciale - Pas de Modification 4.0 International (CC BY-NC-ND) 


\title{
Nommer les biens du Patrimoine mondial : processus de patrimonialisation et réinvention toponymique
}

\author{
Christophe Gauchon
}

\section{Introduction}

1 Le 22 juin 2014, le Comité du patrimoine mondial de l'Unesco annonçait l'inscription de la Grotte ornée du Pont-d'Arc, dite Grotte Chauvet-Pont-d'Arc, Ardèche sur la liste du Patrimoine mondial. Or, en 2007, la France avait déposé ce bien sur sa liste indicative comme "la grotte ornée Chauvet Pont d'Arc ». La longue préparation du dossier de candidature avait donc intégré une évolution toponymique qui peut sembler anecdotique au regard des enjeux d'une inscription sur la liste du Patrimoine mondial. Les conflits avec les inventeurs, les contentieux financiers, les exigences du marketing touristique et territorial avaient amené l'État français à délaisser le nom initial qu'il avait pourtant officialisé dans plusieurs actes, et à en promouvoir un autre. Parallèlement, au printemps 2015, ouvrait la restitution de la grotte Chauvet, équipement touristique qui allait accueillir des centaines de milliers de visiteurs sous le nom de «caverne du Pont d'Arc»; mais quatre ans plus tard, pour la saison 2019, ce nom est modifié et devient "Chauvet 2 », sans que l'on connaisse la part respective des raisons commerciales et juridiques. Ainsi mesure-t-on bien comment les enjeux patrimoniaux et touristiques peuvent concourir à la production néotoponymique. Les multiples interactions entre reconnaissance patrimoniale et valorisation touristique se traduisent ici par des allers-retours entre un référentiel toponymique strict (Pont d'Arc) et un référentiel anthroponymique qui a pris valeur de toponyme (Chauvet).

2 La question ici posée est donc celle du rapport entre patrimonialisation et production toponymique. En quoi le choix du nom participe-t-il à la construction de la valeur 
patrimoniale d'un bien? Les liens entre patrimoine et toponymie sont le plus souvent envisagés à sens unique, les toponymes étant identifiés comme autant d'éléments du patrimoine culturel immatériel (Jullian, 1923; Wydmush, 1998). Ce point de vue a fait l'objet de nombreuses études, y compris sous le parrainage du groupe d'experts des Nations-Unies pour les noms géographiques (Cantile and Kerfoot, 2016). Or il ne s'agit pas seulement de considérer les noms de lieux pour leur valeur patrimoniale, mais aussi de prendre en compte la façon dont la mise en valeur des patrimoines s'accompagne d'une production toponymique.

3 Lorsqu'un espace protégé est créé, il convient toujours de doter le Parc national, la réserve naturelle ou le site classé d'un nom officiel qui figurera dans le décret de création puis dans la nomenclature en usage ; ainsi le Parc national des Écrins, créé en 1973, a-t-il promu un nom resté jusque-là plus confidentiel que Pelvoux ou HautDauphiné mais qui s'est rapidement s'imposé par rapport à eux. Ces noms ont été plus ou moins réfléchis et débattus, ils servent les intérêts de tel groupe, ou bien permettent de gagner l'adhésion de tel autre (Gauchon, 2014). Ces noms ont aussi la particularité de confondre en une seule et même appellation un espace et le statut qui lui est associé, renouant ainsi avec une logique de production toponymique remontant au moyen-âge (Villefranche, Savigné-l'Évêque, Choisy-le-Roi...).

4 En 2019, vingt-huit nouveaux biens, situés dans vingt-cinq pays différents, ont été inscrits par l'UNESCO sur la liste du Patrimoine mondial; un vingt-neuvième, le lac d'Ohrid, a fait l'objet d'une importante extension sur sa rive albanaise. La liste de ces vingt-neuf biens montre une grande diversité des noms par lesquels ils sont désignés : certains sont d'une extrême simplicité (Babylone $\left.)^{1}\right)$, d'autres donnent lieu à des formulations plus compliquées (Parc national de Vatnajökull, la nature dynamique du feu et de la glace), d'autres encore ne comportent aucune mention explicite de leur localisation (Tombes de la culture Dilmun).

5 Et il en va ainsi pour l'ensemble des biens inscrits sur la liste du Patrimoine mondial: dès lors que la Convention du Patrimoine mondial (1972) énonce comme critère d'inscription des biens leur valeur universelle exceptionnelle (notion répétée trois fois dans l'article 1 et trois fois encore dans l'article 2 de la convention), les noms produits peuvent être autant d'arguments forgés dans ce sens. Les 1121 biens inscrits $^{2}$ entre 1978 et 2019 sur la Liste du Patrimoine mondial fournissent un riche corpus qu'il est alors possible d'étudier sous l'angle de la production toponymique : dans quelle mesure ces noms participent-ils de la démonstration de la valeur universelle exceptionnelle? Comment s'articule la relation entre le nom du bien et l'argumentaire qui l'accompagne pour en établir l'authenticité, l'intégrité et la valeur universelle exceptionnelle ? Cet argumentaire vise-t-il toujours à universaliser le sens donné au bien, ou au contraire le spécifie-t-il parfois dans un contexte de concurrence exacerbée ? Le choix du nom rend compte de toute une gamme d'arbitrages entre l'ancrage particularisant et la tension vers l'universel. De plus, le nom est susceptible d'évoluer au cours de la procédure et d'alimenter une forme de prolifération toponymique: que nous apprennent les changements de noms? L'une des difficultés à laquelle s'est heurtée cette recherche réside dans le caractère souvent lacunaire des archives de l'Unesco. Les documents disponibles sur le site du Patrimoine mondial se limitent en général à des relevés de conclusions, et les motivations des différents acteurs ne peuvent être reconstituées qu'indirectement, par exemple en les confrontant aux textes de doctrine qui 
accompagnent et complètent au fil des décennies la Convention de 1972, ou, pour notre cas, aux études générales sur la production néotoponymique.

6 La production néotoponymique fait apparaître deux temps distincts : d'abord le choix du nom sous lequel le bien sera inscrit, avec une large gamme de formulations où le toponyme stricto sensu tient une place très variable (I); puis, dans certains cas, l'évolution de ce nom initial qui répond à un souci de remotivation toponymique (Kristol, 2002) (II). Une fois décryptés les arcanes de cette production toponymique, nous pourrons nous demander si elle sert réellement la valeur universelle exceptionnelle promue par l'UNESCO (III).

\section{Nommer les biens pour construire le patrimoine}

7 La patrimonialisation procède avant tout d'un discours performatif qui vise à conférer à un bien une valeur distinctive et un statut dérogatoire. Ce discours mobilise, entre autres, le registre des toponymes : chaque bien inscrit par l'UNESCO est désigné dans la liste du Patrimoine mondial par un nom. Avant de figurer dans le dossier de candidature, ce nom a fait l'objet d'une réflexion par l'État qui l'a d'abord inscrit sur sa liste indicative. Ce nom doit être cohérent avec l'intention qui a justifié la candidature, avec les critères retenus et avec la déclaration d'authenticité. Le nom est alors bien plus qu'une simple étiquette apposée sur un bien parmi d'autres dans une liste : il contient un message qui, projeté vers l'extérieur, doit donner de la force à une candidature et démontrer le caractère patrimonial du bien et sa valeur universelle exceptionnelle, puisque la demande de l'Unesco est formulée en ces termes.

Ce postulat implique que l'on ne considère pas la toponymie comme un simple héritage dont l'étymologie seule donnerait la clé. L'approche stratigraphique institue le toponyme comme un objet mort, souvenir d'une couche historique ou culturelle plus ou moins enfouie (Zadora-Rio, 2001). Or, les sociétés contemporaines produisent une abondante néotoponymie soit pour désigner de nouveaux lieux (quartiers d'affaires, zones d'activités, villes nouvelles...), soit pour tenir compte de nouveaux contextes géopolitiques ou culturels, soit pour proclamer le changement de statut de tel ou tel lieu (Giraut et Houssay-Holzschuch, 2008). Les toponymes peuvent alors être considérés comme des marqueurs territoriaux actuels et actualisés (Gauchon, 2010). Ils participent d'une volonté assumée d'affichage de l'identité du territoire, soit dans le sens de la conformité à un modèle validé, soit à la recherche d'une singularité qui le distinguera de ses pairs.

9 La «Convention concernant la protection du patrimoine mondial » adoptée en 1972 sous l'égide de l'UNESCO et depuis lors ratifiée par 193 États, prévoit clairement que les biens destinés à figurer sur la Liste sont localisés ; l'article 2 répète à deux reprises que les sites ou les zones seront "strictement délimités", et l'article 3 revient sur la nécessité « d'identifier et de délimiter les différents biens situés sur le territoire [de chaque État] ", ce qui est une condition de leur protection, de leur conservation, de leur mise en valeur et de leur transmission (article 4). Le bien et le lieu où il se situe sont indissociables, et ce lien sera rendu concret par la mise en place de plans de gestion couvrant la zone délimitée. Dès lors, nommer ces biens signifie nécessairement nommer les lieux, et l'on peut considérer que les noms tels qu'ils apparaissent dans la Liste ont valeur de toponymes (Gauchon, 2007). 


\section{Une première typologie des noms retenus peut être esquissée, selon un gradient de recours aux toponymes}

Des biens dont le nom se résume à un seul toponyme, préexistant et employé absolument: ces appellations se retrouvent tout au long des 40 ans d'existence de la Liste, depuis Mesa Verde (reprécisé depuis en Parc national de Mesa Verde) ou Persépolis inscrits sur la Liste respectivement en 1978 et en 1979, jusqu'à Babylone ou Bagan en 2019. De telles appellations, plutôt minoritaires dans l'ensemble, supposent que ces biens bénéficient d'une notoriété suffisante pour se passer de toute précision. Les stratégies de dénomination varient aussi selon les États; au Liban sur les cinq biens inscrits, quatre appartiennent à cette catégorie : Anjar, Baalbek, Byblos et Tyr, sans aucun autre élément d'appréciation, ni de caractérisation objective de la valeur du bien, ni de sa filiation historique ou culturelle, sans nom générique, sans mention de catégorie à laquelle rattacher ces biens. Cette sécheresse d'appellation répond peut-être à un souci de neutralité : le nom de Baalbek paraît suffisamment évocateur pour que l'État libanais n'ajoute pas le problème de l'identifier comme phénicien, romain, islamique ou croisé, puisque de fait il est tout cela à la fois !

11 Des biens dont le nom résulte d'une combinaison de termes spécifiques: le toponyme spécifique est éclairé par la présence d'un autre toponyme plus englobant qui signe à la fois la localisation et l'appartenance : Quartier de «Bryggen » dans la ville de Bergen (1979), Châteaux d'Augustusburg et de Falkenlust à Brühl (1984) ou Sansa, monastères bouddhistes de montagne en Corée (2018). Cette redondance donne d'ailleurs une indication sur la faible notoriété de ces monuments : Angkor ou la statue de la Liberté n'ont pas besoin d'un tel surcroît de précisions. Charge à l'argumentaire de préciser en quoi ces biens peuvent se prévaloir d'une valeur universelle exceptionnelle, ce que les noms, à eux seuls, n'indiquent guère.

Il arrive que le deuxième toponyme soit remplacé par un adjectif qui assume les mêmes fonctions comme dans le Haut lieu tectonique suisse Sardona (2008). Le deuxième terme spécifique n'est pas nécessairement un toponyme, comme dans Les collines du Prosecco de Conegliano et Valdobbiadene (2019), où le nom du vin produit, plus connu que celui des villages, vient expliciter la raison d'être de cette inscription.

Des biens dont le nom se présente comme la combinaison simple d'une catégorie générique et d'un terme spécifique: Ville de Quito ou île de Gorée inscrits en 1978, Cathédrale de Cologne (1996) ou Observatoire de Jodrell Bank (2019). La mention de la catégorie, soit dans une formulation tautologique (car que peuvent être Quito ou Gorée sinon une ville et une île?), soit pour désigner un élément remarquable dans un ensemble plus vaste (Cathédrale de Cologne) vise à indiquer ce qui fait la valeur constitutive du bien ou à en faciliter la remémoration. De tels noms étaient fréquents dans les vingt premières années, même si des locutions descriptives plus élaborées étaient aussi mobilisées à côté des simples catégories génériques, comme pour les Églises creusées dans le roc de Lalibela (1978). Mais ces locutions descriptives n'ont cessé de se complexifier au fil du temps, dans le but de mieux faire valoir la spécificité de chaque bien pour en marquer l'originalité. La catégorie générique devient alors partie prenante de l'argumentation, comme dans: Complexe industriel de la mine de charbon de Zollverein à Essen (2001), Lagons de Nouvelle-Calédonie: diversité récifale et écosystèmes associés (2008) ou Paysage d'élevage et de dressage de chevaux d'attelage cérémoniels à Kladruby nad Labem (2019). L'équilibre des termes, dans ce dernier exemple surtout, 
suggère que la nature du bien telle qu'elle est détaillée prime sur sa localisation, le toponyme n'apportant qu'une précision complémentaire. Ici, en s'appuyant sur un toponyme spécifique préexistant, c'est la combinaison des termes spécifiques et génériques qui crée une nouvelle appellation et qui fonctionne comme un néotoponyme s'appliquant au lieu tel qu'il est patrimonialisé par son inscription sur la Liste. La mine de Zollverein ou les lagons de Nouvelle-Calédonie ne sont plus seulement désignés pour eux-mêmes mais pour leurs qualités éminentes qui leur valent cette inscription.

Des biens enfin dont les noms se présentent sans aucun ancrage toponymique ou avec un ancrage très lâche : dès les années 1980 étaient apparus sur la liste des noms qui référaient à un complexe culturel non situé, comme Bâtiments traditionnels ashanti (1980) ou La culture chaco (1987). Dès 1997, l'Italie avait porté la candidature des Résidences des Savoie, Savoie ne désignant pas ici la région mais la dynastie à l'origine de la création du royaume d'Italie en 1861. Puis le sultanat d'Oman fit inscrire coup sur coup le Sanctuaire de l'oryx arabe en 1994 (rayé de la Liste en 2007) et la Terre de l'encens en 2000, tous deux sans référence à une quelconque localisation. Plus récemment, deux biens ont été inscrits sur la Liste : L'ouvre architecturale de Le Corbusier, une contribution exceptionnelle au Mouvement Moderne (2016) et Les cuvres architecturales du XXe siècle de Frank Lloyd Wright (2019).

Ces noms génériques peuvent être accompagnés d'un toponyme qui donne une indication extrêmement large sur leur localisation, comme les Forêts primaires et anciennes de hêtres des Carpates et d'autres régions d'Europe (nom actuel après deux extensions successives du bien) ou Sites palaffitiques préhistoriques autour des Alpes (2011), sachant que dans ce cas, "autour des Alpes " s'étend aussi au Jura français, suisse et souabe... De tel noms présentent l'avantage de ne pas figer la délimitation du bien, qui peut être étendu à des forêts de hêtres, à des régions de culture de l'encens ou à des éléments de l'œuvre architecturale situés hors du ou des pays qui avaient été à l'origine de la candidature. On est ici fondé à s'interroger sur le statut réel de ces noms : sont-ils vraiment des toponymes? Un toponyme peut-il être forgé sur le seul arrangement de noms communs et de termes génériques? En toponymie historique, cela rejoint l'exemple bien connu de l'URSS, nom de pays qui ne référait à aucun espace particulier mais à une forme d'organisation politique.

Une telle dissociation entre le bien et le lieu reste très minoritaire dans la Liste, mais illustre une perméabilité croissante entre le Patrimoine mondial et le Patrimoine culturel immatériel qui font l'objet de deux conventions distinctes. Les architectures de Le Corbusier et de Wright ne sont pas intangibles, mais la dénomination des biens indique clairement que ce sont les œuvres de l'esprit qui sont patrimonialisées autant que les réalisations elles-mêmes. L'absence de toponyme spécifique peut même militer pour la valeur universelle du bien, et rejoint sous certains aspects les noms des pratiques inscrits sur la Liste du patrimoine immatériel.

$16 \mathrm{Au}$ regard de la toponymie, le statut des biens inscrits sur la Liste du patrimoine mondial n'est donc pas homogène. Mais ce statut est lié aussi à la configuration spatiale du bien inscrit. Sans qu'il soit question de faire correspondre strictement les catégories, les biens inscrits sur la Liste du Patrimoine mondial s'organisent selon quatre modèles spatiaux : des localisations ponctuelles pour un monument isolé (logique de site); des régions plus ou moins vastes, souvent lorsqu'il s'agit d'un paysage culturel, comme le Paysage culturel de la Wachau en Autriche; des biens qui s'organisent le long de routes ou 
d'axes variés, comme Qhapaq Ñan, réseau de routes andin (2014); et des biens dits sériels dont l'inscription crée une logique réticulaire. Or ce sont ces biens sériels, dont la localisation est moins contrainte et dont la liste résulte d'un processus de sélection souvent complexe, qui sont parfois dotés de noms sans dimension toponymique explicite, comme Le jardin persan (2011) ou les Cimetières de tombes médiévales stećci (2019, bien transfrontalier concernant quatre anciennes républiques yougoslaves).

\section{Changer les noms pour remotiver la patrimonialisation}

Les demandes de changements de noms sur lesquelles le Comité du patrimoine mondial statue lors de ses sessions annuelles prouvent l'importance accordée à la dénomination des biens dans le processus de patrimonialisation : en 2006, le Comité examina dix-huit demandes de changements de noms de biens déjà inscrits, émanant de sept pays différents (tableau 1 en annexe); en 2007, il a encore approuvé huit changements de noms dans cinq pays différents ; et en 2013, il en a approuvé sept autres, prouvant parlà que la Liste n'est pas stabilisée sur le plan toponymique. Les États demandeurs de ces changements expriment ainsi le souci que les noms figurant sur la liste correspondent bien à leurs intentions patrimoniales du moment. Ces changements peuvent intervenir dans différentes circonstances :

\section{Changement de nom au cours de la procédure d'inscription}

Entre le moment où un État annonce son intention de faire inscrire un bien sur sa liste indicative et le dépôt de la candidature auprès de l'Unesco, il se passe souvent une dizaine d'années, parfois plus. Dans cet intervalle, la construction de la valeur patrimoniale se poursuit : études diligentées pour affiner la connaissance objective du bien, sensibilisation des populations et des acteurs du territoire pour les préparer et les mobiliser en vue de l'arrivée du label tant convoité, mise à niveau des protections règlementaires en conformité avec la législation de l'État partie, finalisation des mesures de gestion, prise en compte des avis des experts internationaux... Le nom du bien a donc le temps de changer plusieurs fois au cours de ce processus. Ces évolutions ne sont pas toujours bien documentées; on peut en relever certaines, mais sur leurs véritables raisons, on ne peut guère que conjecturer à partir de quelques exemples :

Depuis 1996, la liste indicative de la France comportait un bien dénommé «Albi: ensemble urbain de briques, cathédrale, Palais de la Berbie, Pont sur le Tarn ». En 2010, le bien fut inscrit comme Cité épiscopale d'Albi. Ce nouveau nom gagnait en concision, il évitait la mention d'une énième cathédrale sur la liste des biens français et il mettait mieux en évidence la cohérence du bien ${ }^{3}$. Quelques mois plus tard, le 17 mai 2011, la commune d'Albi déposait le nom "Albi : la cité épiscopale » comme marque auprès de l'Institut National de la Propriété Industrielle (INPI) pour toute une série de biens et de services (classes 1 à 45 dans la nomenclature). Ainsi les termes de "cité épiscopale " associés au nom d'Albi bénéficient-ils d'une double promotion, l'une d'ordre symbolique (l'inscription par l'Unesco), l'autre plus strictement économique (la marque déposée), et toutes les deux convergent autour d'un nom érigé comme nouvel emblème brandi par la cité tarnaise. 


\section{Changement de nom à l'occasion d'une extension du bien} Olga) avait d'abord été inscrit en 1987 comme bien naturel. En 1994, en glissant vers la catégorie "paysage culturel », il devient Parc national d'Uluru-Kata Tjuta, le changement de catégorie et de nom marquant un travail de réappropriation du site par la culture aborigène : Ayers Rock, le nom anglo-saxon du fameux monolithe de grès rouge, a ainsi disparu de la liste, laissant la place à un nom rendant mieux compte de la place de ce géosymbole dans la cosmogonie aborigène. Idem pour SGGang Gwaii au Canada, dont le toponyme colonial (île Anthony) jusque-là inscrit entre parenthèses, disparaît en 2006. 
À un autre stade de la prise en compte des toponymes locaux, l'Ensemble archéologique de la vallée de Boyne (Irlande), inscrit en 1999, a été renommé en 2013 Brú na Bóinne Ensemble archéologique de la vallée de Boyne pour mettre en avant le nom gaélique. Cette évolution rejoint le mouvement de restitution toponymique à l'œuvre dans de nombreuses parties du monde (Giraut et al., 2008) et dont la patrimonialisation est un des moyens de propagation.

\section{Comment (re)nommer Auschwitz?}

Lorsqu'en 1979, la Pologne fit inscrire sur la liste du Patrimoine mondial le Camp de concentration d'Auschwitz, elle déposa à dessein le nom allemand et non pas le nom polonais d'Oswiecim. Pour rappel, Oswiecim se trouvait en Pologne avant septembre 1939 et avait été annexée après septembre 1939 à l'Allemagne (province de HauteSilésie), d'où la germanisation du toponyme ; cette dissociation d'avec le nom local et l'emploi intentionnel d'un exonyme sont exceptionnels dans la Liste et marquent une volonté évidente de mise à distance par l'État-partie à l'origine de l'inscription, i.e. la Pologne. Ce recours à un exonyme est d'autant plus notable qu'un souci de "polonisation" du souvenir était en même temps observé dans le processus de muséification du camp (Szurek, 1990). Mais la mémoire de la seconde guerre mondiale a tellement installé Auschwitz dans la conscience humaine que Camp de concentration d'Auschwitz, dans sa forme ramassée, traduisait mieux qu'Oswiecim la signification universelle exceptionnelle de ce bien.

En 2006, le Comité du patrimoine mondial examina la requête de l'État polonais qui visait à changer le nom du bien pour "Ancien camp de concentration allemand nazi d'Auschwitz-Birkenau ", l'approuva sur le principe mais subordonna sa décision à l'affirmation des programmes éducatifs autour de l'histoire d'Auschwitz. L'année suivante, il admit la transformation du nom qui devint: Auschwitz-Birkenau, avec comme sous-titre camp allemand nazi de concentration et d'extermination, 1940-1945. Cette appellation est exceptionnellement complexe et précise au regard de la multiplicité des champs sémantiques sollicités; dans la liste de l'Unesco, il s'agit du seul bien qui se voit attribué un sous-titre.

Le nom proposé en 2006 puis le nouveau nom adopté en 2007 vont dans le sens d'une spécification plus forte en identifiant explicitement les bourreaux sur un double plan national et idéologique (allemand nazi) d'où une mise à distance encore plus marquée du point de vue polonais, et en cadrant la période chronologique concernée. Le premier nom, même s'il était en partie inexact sur le plan historique (le terme de Camp de concentration ne rendait pas compte de la réalité propre d'Auschwitz), tendait beaucoup plus vers l'universalité en mettant en avant le seul nom d'Auschwitz sans qu'il soit nécessaire de préciser davantage. Le nom validé en 2007 est historiquement plus rigoureux (Auschwitz-Birkenau et camp de concentration et d'extermination) mais au risque de diluer la force de l'expression initiale.

Bien sûr, la dimension toponymique ne constitue qu'un des aspects de la mise en mémoire complexe et souvent conflictuelle d'Auschwitz. Ce cas tout à fait particulier montre les enjeux attachés au choix de l'appellation et à sa réévaluation à trente ans d'intervalle (1945-1979-2007), en fonction des événements politiques, historiographiques et mémoriels qui se sont déroulés dans l'intervalle. 


\section{À travers les noms du Patrimoine mondial, la réalité de la valeur universelle?}

29 Nous avons cité supra les noms de quatre biens inscrits par le Liban, pour leur sécheresse d'énonciation: Anjar, Baalbek, Byblos et Tyr. Mais, à l'opposé, le cinquième bien libanais, inscrit après la fin de la guerre civile, en 1998, s'intitule de façon complexe et polyglotte : Ouadi Qadisha ou Vallée sainte et forêt des cèdres de Dieu (Horsh Arz el-Rab); le nom associe deux toponymes spécifiques transcrits de l'arabe et des catégories descriptives qui fonctionnent aussi comme des traductions partielles des noms arabes. Ce nom ne rattache la valeur patrimoniale du bien à aucune communauté particulière, alors que son inscription le désignait manifestement comme haut lieu des Maronites (Pasquier, 2011). Mais le dispositif spatial retenu et les enjeux culturelsconfessionnels-communautaires, trois adjectifs largement synonymes au Liban, ne permettaient pas de désigner ce bien par une formule aussi laconique que les quatre autres.

30 De nombreux débats ont essayé de définir au plus proche la Valeur exceptionnelle universelle; si l'on se réfère à la déclaration des experts réunis en 2005 par l'ICOMOS ${ }^{7}$ : "Valeur universelle signifie qu'un monument, site ou ensemble a une valeur qui dépasse la valeur locale ou régionale pour atteindre une valeur qui peut être considérée comme universelle $»^{8}$. Et plus loin: "Pour déterminer si un bien a une valeur universelle exceptionnelle, il faut : $a$. définir ses qualités, $b$. considérer la valeur de ses qualités, c. considérer si cette valeur est locale, régionale ou universelle ${ }^{9}$. Autrement dit, "la valeur universelle exceptionnelle signifie une importance culturelle et/ou naturelle tellement exceptionnelle qu'elle transcende les frontières nationales et qu'elle présente le même caractère inestimable pour les générations actuelles et futures de l'ensemble de l'humanité $\aleph^{10}$. Dans ce texte, il est patent que l'universalité dépend du caractère hautement exceptionnel du bien; les deux adjectifs « universelle exceptionnelle » ne sont donc pas sur le même plan. L'universalité résulte d'une somme de caractéristiques convergentes, et justifie que «l'humanité tout entière » (aux termes de la convention de 1972) s'implique dans la préservation de ce patrimoine.

31 Le croisement de ces deux définitions montre qu'une distinction doit être opérée entre une valeur strictement locale ou régionale, et une valeur locale ou régionale associée à une portée universelle. Les biens culturels s'inscrivent d'abord dans une culture locale, régionale ou nationale avant d'acquérir une portée plus générale, voire universelle.

\section{Des noms mis au service des particularismes?}

Chaque État élabore ses propres stratégies à la fois dans le choix des biens à faire inscrire et dans la façon de les dénommer, les deux se combinant pour parvenir à la patrimonialisation la plus efficace. Le passage en revue de la liste du Patrimoine mondial livre quelques appellations qui semblent miser sur les particularismes plus que sur l'universalité. Ces noms sont minoritaires mais l'on peut ainsi distinguer :

- des noms qui mettent en avant la dimension nationale, en mentionnant soit le nom du pays, soit un adjectif de nationalité : Paysage culturel du café de la Colombie (2011); Villages historiques de Corée : Hahoe et Yangdong (2010) ou Haut lieu tectonique suisse Sardona (2008). Dans ce dernier cas, déjà cité, on s'étonne de l'association d'un adjectif de nationalité avec la mention d'un phénomène géologique... Lors de sa session de 
juillet 2019, le Comité du patrimoine mondial inscrit un bien naturel au nom fort contourné : Sanctuaire d'oiseaux migrateurs le long du littoral de la mer Jaune et du golfe de Bohai de Chine. Cette ultime précision est surprenante, puisque nul ne conteste que le golfe de Bohai soit entièrement en Chine et elle semble donc complètement redondante; mais cette redondance légitimatrice rejoint des usages observables dans le langage courant, comme lorsque l'on parle du «Valais suisse ».

- des noms qui s'ancrent dans une période historique particulière : Palais impériaux des dynasties Ming et Qing à Beijing et à Shenyang (1987) ou Résidences des Savoie (1997) ou Les Lombards en Italie. Lieux de pouvoirs (568-774 ap. J.C.) (2011). Le fait d'associer le bien à une période particulière de l'histoire ne peut pas masquer la dimension nationale sous-jacente. Dans le même registre, la Chine a ainsi fait inscrire le Mausolée du premier empereur Qin, les Tombes impériales des dynasties Ming et Qing, le Palais d'été, Jardin impérial de Beijing et le Temple du ciel, autel sacrificiel impérial à Beijing : soit pas moins de cinq biens, inscrits entre 1987 et 2000, dont les noms réfèrent explicitement au passé impérial de la Chine! Sous couvert de valeur universelle exceptionnelle, la Liste du Patrimoine mondial est ainsi mise au service de l'exaltation de l'histoire de l'État partie.

- des noms qui renvoient à une dimension ethnique ou culturelle : ils semblent moins nombreux. On peut citer les Bâtiments traditionnels ashanti, au Ghana (1980), qui évoquent à la fois une composante du peuplement et une période historique (XVIII ${ }^{\mathrm{e}}$ $\mathrm{XIX}^{\mathrm{e}}$ siècles), ou les Sites Gusuku et biens associés du royaume des Ryukyu, au Japon (2000), le terme Gusuku faisant référence à des communautés paysannes des $\mathrm{XI}^{\mathrm{e}}$ et $\mathrm{XII}^{\mathrm{e}}$ siècles, ou les Anciennes cités pyu, au Myanmar (2014). La liste du Patrimoine mondial montre ainsi un fort penchant pour la culture des particularismes, à l'échelle du pays ou de la région. Le dossier d'inscription des Églises romanes catalanes de la vallée de Boi est révélateur de cette tendance. Le nom retenu, avec le double adjectif, roman (style architectural) et catalan (ancrage territorial culturel), rappelle que le bien a été proposé par la Communauté Autonome de la Catalogne, comme le prévoit l'article 148 de la constitution espagnole (1978) définissant les domaines de compétences des régions et comme le précise le dossier d'inscription : «l'ensemble de la société civile et les institutions de la Catalogne ont donné leur consentement unanime à la demande d'inscription de la Vall de Boi en tant que Patrimoine mondial »; l'État espagnol a porté le bien sur sa liste indicative en 1998 et en a obtenu l'inscription sur la liste de l'Unesco en 2000. Les "églises romanes catalanes" font ainsi écho au Palais de la musique catalane et hôpital de Saint-Pau de Barcelone, inscrits dès 1997.

\section{Dans quelle langue désigner les biens?}

Au-delà du signifié, la langue est également mobilisée pour faire valoir l'ancrage des éléments du patrimoine. Lorsqu'une région, un lieu ou un bien sont désignés par une pluralité de noms dans différentes langues, l'État porteur de la candidature fait le choix d'un nom plutôt que d'un autre. Les appellations bi- ou plurilingues sont rares et se raréfient au bénéfice des seuls toponymes précoloniaux (cf. supra).

Ce choix comporte une dimension linguistique et, au-delà, participe de l'appropriation du bien : depuis son identification au milieu du XIX ${ }^{e}$ siècle, le mont Everest a été connu sous au moins huit noms différents, sans compter les variantes de graphie ni les erreurs d'identification, et le nom retenu par l'administration impériale britannique a été contesté dès l'origine. Dans les années 1960, le gouvernement népalais a souhaité 
promouvoir un nom proprement népalais et inventa alors le nom de Sagarmatha pour désigner à la fois le massif et le point culminant lui-même (Unsworth, 1986). La pertinence de ce nom a été discutée, mais lorsque le Népal déposa la candidature au Patrimoine mondial, le dossier fut présenté sous le titre : Parc national de Sagarmatha. Les publications associées à l'Unesco désignent donc désormais le toit du monde sous le nom officiel retenu en 1979 au motif que «pour les Népalais, ce sommet incarne la déesse Sagarmatha » (Amaro, 1997).

L'éviction du nom tibétain de Chomolungma, pourtant attesté depuis bien plus longtemps, a été facilitée par le fait que le bien est inscrit dans des limites strictement népalaises sans dimension transfrontalière avec la Chine-Tibet. La présence de Sagarmatha sur la liste de l'Unesco évince à la fois le toponyme colonial plus identifié sur le plan international et le toponyme tibétain. Le nom promu vise donc à valoriser une approche nationale particularisante.

Il en va à peu près de même pour l'île de Pâques dont le paysage ponctué de grandes statues monolithes a été inscrit en 1995 sous le nom de Parc national de Rapa Nui, «nom autochtone de l'île de Pâques ", de telle sorte que la liste présente une forme de dédoublement toponymique, la langue des Pascuans étant utilisée non pas pour désigner l'île elle-même mais le paysage culturel qui fait patrimoine. Cette inscription, dans ces termes, rejoint la question de la toponymie comme support d'affirmation des cultures autochtones.

En 2019, à la demande de l'Ukraine, le Comité du patrimoine mondial a accepté que Kiev devienne Kyiv. Au contraire, la Jordanie avait obtenu en 1981 l'inscription de la Vieille ville de Jérusalem et ses remparts, dans la graphie habituellement retenue en Europe, perçue comme plus neutre, et non pas dans la transcription du nom arabe (Al-Quds), ni hébreu (Yerushalàyim).

\section{Conclusion}

Certains noms évoluent donc en gommant certains particularismes et tendent vers une plus grande universalité : ainsi le Mur d'Hadrien, inscrit par le Royaume-Uni dès 1987 at-il été étendu en 2005 à l'Allemagne sous la nouvelle appellation des Frontières de l'empire romain. Ce nom permet d'affranchir le bien d'une localisation particulière et d'intégrer un ensemble civilisationnel plus vaste; ni l'ancien nom, ni le nouveau ne comportent d'ailleurs de référence toponymique explicite.

D'autres noms illustrent le souci de présenter des candidatures originales sans redondance avec des biens déjà inscrits, ce qui pousse les États à cultiver des formulations toujours plus particularisantes. Ainsi en 2015, la France a fait inscrire sur la Liste le vignoble de Champagne sous le nom sibyllin de Coteaux, Maisons et Caves de Champagne, prolongeant ainsi la veine amorcée dès 1999 avec la Juridiction de SaintEmilion. Alors que Les climats du vignoble de Bourgogne (2015), le Paysage viticole de l'île du Pico (Açores, Portugal, 2004) et Lavaux, vignobles en terrasses (Suisse, 2007) se donnent plus clairement pour ce qu'ils sont, Champagne et Bordelais avancent masqués et jouent la carte de la spécificité par rapport aux vignobles déjà inscrits. Toutefois, le recours à la métonymie (Debarbieux, 1999), les «maisons et caves » ou la "juridiction » pour désigner le paysage culturel organisé autour des vignobles, s'il peut flatter les 
sociétés locales, vise à sur-singulariser ces biens par rapport aux autres de la même catégorie et ne paraît pas de nature à faire valoir l'universalité de ces patrimoines.

Mais dans les faits, la tentation est forte d'utiliser le patrimoine pour fonder ou renforcer une démarche identitaire, à l'échelle des États ou à une échelle infra-étatique. D'où l'émulation entre les pays pour avoir le plus de biens inscrits sur la Liste ou, pour des micro-États, d'y faire figurer au moins un bien (Andorre, les Fidji ou la Dominique, par exemple); d'où la faible proportion de biens transfrontaliers qui ne favorisent pas ce type d'appropriation; d'où l'effort pour estampiller des biens comme catalans, wallons ou lombards. Les noms peuvent aussi être choisis pour leur dimension territoriale et apparaissent comme autant de gages pris sur le règlement de conflits d'appropriation, par exemple lorsque la Roumanie a fait inscrire en 1993 un bien appelé Eglises de Moldavie ; ou lorsque la Serbie obtient en 2006 l'extension du bien Monastère de Dečani (d'abord inscrit en 2004) en y ajoutant trois autres églises ou monastères qui deviennent dès lors Monuments médiévaux au Kosovo ${ }^{11}$ ! On peut s'étonner que l'Unesco, agence de l'ONU, valide de telles appellations qui valent revendication géopolitique et remise en cause des frontières.

Le discours du patrimoine est un des moyens par lesquels se déploie la politique culturelle des États ; la politique toponymique, même si elle n'est pas toujours pensée comme telle, en est le prolongement. La liste du patrimoine mondial fournit de nombreux exemples de cette réinvention toponymique qui consacre, aux côtés des noms patinés par l'usage, des noms expressément construits à des fins de patrimonialisation. Plusieurs biens inscrits sur la liste du Patrimoine mondial ont ainsi déjà eu trois noms différents. Ces pratiques engendrent une insécurité toponymique par la succession d'appellations susceptibles de se remplacer les unes les autres au gré des choix politiques, au lieu de s'installer dans le temps long des toponymes, rejoignant ainsi les mutations toponymiques qui accompagnent parfois les changements de régimes politiques. Demeure alors la question de l'usage de ces néotoponymes, de leur diffusion, et de leur capacité à s'imposer par rapport à des toponymes préexistants...

\section{BIBLIOGRAPHIE}

Amaro C., 1997. Un trekking au Sagarmatha. Revue du Patrimoine mondial, n 5, p. 7.

Cantile A., Kerfoot H., 2016. Place names as intangible cultural heritage. IGMI Florence, 223 p.

Cristol A.M., 2002. Motivation et remotivation des noms de lieux : réflexions sur la nature linguistique du nom propre. Rives méditerranéennes, $\mathrm{n}^{\circ}$ 11, p. 105-120.

Debarbieux B., 1995. Le lieu, le territoire et trois figures de rhétorique. L'espace géographique, $\mathrm{n}^{\circ} 2$, p. 97-112.

Gauchon C., 2007. Territoires touristiques et néo-toponymie : les noms des grands domaines skiables des Alpes françaises. Loisir et Société/Society and Leisure, vol. 30, n 1, p. 69-88. 
Gauchon C., 2010. Tourisme et patrimoines : un creuset pour les territoires ?, mémoire d'HDR, Université de Savoie, 211 p. URL : http://tel.archives-ouvertes.fr/docs/00/55/93/70/PDF/ HDR_Gauchon_Christophe_Texte_synthA_se.pdf

Gauchon C., 2014. L'innovation toponymique comme modalité de labellisation des territoires, l'exemple du PNR des Baronnies provençales. In Labellisation et mise en marque des territoires, Clermont-Ferrand, CERAMAC nº 34, p. 233-245.

Giraut F., Houssay-Holzschuch M., Guyot S. 2008. Au nom des territoires ! Enjeux géographiques de la toponymie. L'Espace géographique, $\mathrm{n}^{\circ}$ 2, p. 97-105.

Giraut F., Houssay-Holzschuch M., 2008. Néotoponymie : formes et enjeux de la dénomination des territoires émergents. L'Espace politique, $\mathrm{n}^{\circ} 2$.

Jullian C., 2015 [1 1 ère ed. 1923]. Ne touchez pas aux noms des rues, des Malassis, 48 p.

Pasquier J., 2011. Processus de patrimonialisation des sites religieux dans les espaces protégés de montagne : la Grande Chartreuse (Préalpes du Nord) et la vallée de la Qadisha-forêt des Cèdres du Dieu (Nord-Liban), Thèse géographie. Université de Savoie, 413 p.

Szurek J.-C., 1990. Le camp-musée d'Auschiwtz. In Brossat A., Combe S., Potel J.-Y., Szurek J.-C. (dir.), À l'Est, la mémoire retrouvée, La Découverte, p. 535-565.

Unsworth W., 1985. L'Everest. Ed. fr. Denoël, 486 p.

Wydmusch S. (dir.), 1998. La toponymie, un patrimoine à préserver. L'Harmattan, 176 p.

Zadora-Rio E., 2001. Archéologie et toponymie : le divorce. Les Petits Cahiers d'Anatole, $\mathrm{n}^{\circ} 8$, Université de Tours, $17 \mathrm{p}$.

\section{NOTES}

1. Dans ce chapitre, nous avons écrit en italiques les noms des biens tels qu'ils figurent (ou tels qu'ils ont figuré lorsqu'il y a eu changement) dans la liste de l'Unesco. En revanche, nous avons laissé «entre guillemets" les noms tels qu'ils figurent sur les listes indicatives. Seul le nom français est cité ici, mais nous avons systématiquement vérifié que le nom anglais n'apportait pas de nuance significative, les biens étant inscrits dans ces deux langues. Les documents de l'Unesco disponibles en ligne, les comptes rendus des sessions annuelles relèvent scrupuleusement les décisions prises, mais fournissent peu d'indications sur leurs motivations.

2. 1123 en réalité, si l'on tient compte des deux biens qui ont été rayés de la Liste en 2007 et 2009.

3. On note que le bien «Centre historique d'Avignon » a connu l'évolution exactement inverse (cf. tableau 1en annexe).

4. $30^{\text {ème }}$ session du Comité du atrimoine mondial, Vilnius, décision 30 COM 8B.6.

5. $31^{\text {ème }}$ session du Comité du patrimoine mondial, Christchurch, décision 31 COM 8B.2.

6. $37^{\text {ème }}$ session du Comité du patrimoine mondial, Phnom Penh, décision 37 COM 8B.1.

7. ICOMOS: Conseil international des Monuments et des sites, organisation nongouvernementale qui participe à l'expertise des biens du Patrimoine mondial.

8. Le concept de valeur universelle exceptionnelle, Conseil international des monuments et des sites, Icomos, Kazan, 6-9 avril 2005, p. 26.

9. Idem, p. 28.

10. Orientations devant guider la mise en œuvre de la Convention du patrimoine mondial, 2 février 2005 , p. 15 , § 49 . 
11. Au $1^{\text {er }}$ janvier 2019, le Kosovo n'est pas membre de l'UNESCO ni État partie à la convention du Patrimoine mondial ; ce bien reste donc sur la liste de la Serbie.

\section{RÉSUMÉS}

L'article interroge les noms des biens inscrits par l'Unesco sur la liste du Patrimoine mondial. L'analyse du corpus constitué par ces 1123 noms permet d'en proposer une première typologie selon leur niveau d'ancrage à des toponymes préexistants. Les changements de nom, au cours du processus d'inscription ou une fois cette inscription acquise, renseignent aussi sur l'intention des États parties et sur la façon dont ils se représentent les valeurs de la patrimonialisation. L'analyse de ces noms peut ensuite être confrontée aux textes de doctrine produits par l'Unesco sur la valeur universelle exceptionnelle. Dans quelle mesure les noms proposés participent-ils, ou non, de la démonstration de cette valeur universelle exceptionnelle ? Ou bien ces noms rattachent-ils ces biens à une appartenance historique, nationale ou ethnique, ce qui les particularise au lieu de les projeter vers l'universalité promue par l'Unesco?

Naming the World heritage and characterizing the outstanding universal value. The paper deals with the naming of World Heritage sites as they are listed by UNESCO, as a form of semantic production. The names of the 1123 inscribed sites in 2019 are here analyzed, and a first typology is submitted. Sometimes, the names of the inscribed sites are modified either during the application or several years after their inscription. These changes show the will of members states of individualizing their own heritage, using native languages or imaginative names. Then, the analysis of these names could be compared with the doctrinal texts published by Unesco about the significance of Outstanding universal value. How the selected names can take part in making the proof of this Outstanding universal value? In the whole list, many names can be found with historic, national or ethnic mentions which aim at specific character more than universality promoted by Unesco.

\section{INDEX}

Mots-clés : patrimoine mondial, production toponymie, rhétorique patrimoniale, valeur universelle exceptionnelle

Keywords : World Heritage, production of place names, heritage rhetoric, outstanding universal value

Thèmes : Sur le Champ - Sur le Terrain

\section{AUTEUR}

\section{CHRISTOPHE GAUCHON}

Christophe Gauchon, christophe.gauchon@univ-savoie.fr, est Professeur à l'Université Savoie Mont Blanc-laboratoire Edytem. Il a récemment publié :

- Duval M., Gauchon C., Malgat C., 2020. Construction de l'authenticité et expérience 
patrimoniale : conception et réception de la réplique de la grotte Chauvet-Pont-d'Arc. Culture \& Musées [En ligne], n 35. URL: http://journals.openedition.org/culturemusees/5041 - DOI:

https://doi.org/10.4000/culturemusees.5041

- Gauchon C., 2020. Espaces protégés et territoires labellisés : jusqu'où étendre les périmètres classés ? In Tanchoux P., Priet F. (dir), Les labels dans le domaine du patrimoine culturel et naturel. Presses Universitaires de Rennes, p. 369-380.

- Christophe Gauchon, 2020. Friches (notice), Karst et milieux souterrains (notice), Stations de ski (notice). In Collectif, Dictionnaire critique de l'Anthropocène. Ed. du CNRS. 